\title{
ON ORDERS OF DIRECTLY INDECOMPOSABLE FINITE RINGS
}

\section{YasUYuki Hirano aNd Takao SUmiYama}

Let $R$ be a directly indecomposable finite ring. Let $p$ be a prime, let $m$ be a positive integer and suppose the radical of $R$ has $p^{m}$ elements. Then we show that $p^{m+1} \leqslant|R| \leqslant p^{m^{2}+m+1}$. As a consequence, we have that, for a given finite nilpotent ring $N$, there are up to isomorphism only finitely many finite rings not having simple ring direct summands, with radical isomorphic to $N$. Let $R^{*}$ denote the group of units of $R$. Then we prove that $(1-1 / p)^{m+1} \leqslant\left|R^{*}\right| /|R| \leqslant 1-1 / p^{m}$. As a corollary, we obtain that if $R$ is a directly indecomposable non-simple finite $2^{\prime}$-ring then $|R|<\left|R^{*}\right||\operatorname{Rad}(R)|$.

Stewart [7] considered the following problem. Given a finite group $G$, what are the possible finite rings with group of units isomorphic to $G$ ? In this paper, we consider a similar problem; given a finite nilpotent ring $N$, what are possible finite rings with radical isomorphic to $N$ ? For (not necessarily finite) algebras, this problem was considered by Flanigan [2] and Hall [3].

All the rings considered in this paper are finite, and have an identity. Let $R$ be a directly indecomposable finite ring. Then, as is well known, the order $|R|$ of $R$ is a power of a prime $p$. Let $\operatorname{Rad}(R)$ denote the (Jacobson) radical of $R$ and suppose that $|\operatorname{Rad}(R)|=p^{m}>1$. Mainwaring and Pearson [4] proved that there are at most $m+1$ minimal ideals in $R / \operatorname{Rad}(R)$. Using their method, we try to estimate $|R|$.

For a prime $p$ and positive integers $n$ and $t, G R\left(p^{n}, t\right)$ denotes the Galois extension of $Z / Z p^{n}$ of degree $t$ (see McDonald [5, p.307]). Especially the field $G R(p, t)$ of $p^{t}$ elements is denoted by $G F\left(p^{t}\right)$.

A graph means a finite undirected graph without loops. The edge which joins two vertices $x$ and $y$ is denoted by $(x, y)$.

We begin with the following lemma.

LEMMA 1. Let $G=(V, E)$ be a non-trivial connected graph, where $V$ is the set of vertices of $G$ and $E$ the set of edges of $G$. Let $u$ be a vertex in $V$. Then there exists an injective mapping $\varphi: V \backslash\{u\} \rightarrow E$ satisfying the conditions: (i) $\varphi(w)=(w, u)$ for some $w \in V \backslash\{u\}$, and (ii) for any $v \in V \backslash\{u\}$, one of the endpoints of $\varphi(v)$ is $v$.

Proof: We proceed by induction on $|V|$. In case $|V|=2$, our assertion is trivial. Assume $|V|>2$ and let $G-u$ denote the subgraph of $G$ obtained by deleting the

Received 1 November 1991

Copyright Clearance Centre, Inc. Serial-fee code: 0004-9729/92 \$A2.00+0.00. 
vertex $u$ and all edges incident with $u$. Let $G_{1}=\left(V_{1}, E_{1}\right), \ldots, G_{r}=\left(V_{r}, E_{r}\right)$ be the connected components of $G-u$. Then, for each $i$, there exists $u_{i} \in V_{i}$ such that $\left(u, u_{i}\right) \in E$. If $G_{j}$ is non-trivial, namely $\left|V_{j}\right|>2$, then by induction hypothesis there exists an injective mapping $\varphi_{j}: V_{j} \backslash\left\{u_{j}\right\} \rightarrow E_{j}$ satisfying the conditions (i) and (ii). We now define a mapping $\varphi: V \backslash\{u\} \rightarrow E$ by $\varphi\left(u_{j}\right)=\left(u, u_{i}\right)$ for each $i$ and $\varphi(v)=\varphi_{j}(v)$ if $v \in V_{j} \backslash\left\{u_{j}\right\}$. It is easy to see that $\varphi$ satisfies (i) and (ii).

It is easy to check the set

$$
\left\{\left(a_{i j}\right) \in M_{m+1}(G F(p)) \mid a_{21}=a_{31}=\ldots=a_{m+1,1}=0\right\}
$$

forms a subring of $M_{m+1}(G F(p))$. We denote this subring by $A_{m+1}(p)$.

We shall estimate the order of a non-simple directly indecomposable finite ring in terms of the order of its radical.

TheOREM 1. Let $R$ be a directly indecomposable finite ring and suppose $|\operatorname{Rad}(R)|$ $=p^{m}$ where $p$ is a prime and $m$ is a positive integer. Then

$$
p^{m+1} \leqslant|R| \leqslant p^{m^{2}+m+1} .
$$

The first equality holds if and only if $R / \operatorname{Rad}(R)=G F(p)$, and the second equality holds if and only if $R$ is either isomorphic or anti-isomorphic to $A_{m+1}(p)$.

Proof: Since $R$ has 1 , we have $p \leqslant|R / \operatorname{Rad}(R)|$, so that $p^{m+1} \leqslant|R|$. The equality holds if and only if $R / \operatorname{Rad}(R)=G F(p)$.

To prove the latter inequality, suppose that $R / \operatorname{Rad}(R)=M_{n_{1}}\left(K_{1}\right) \oplus \ldots \oplus$ $M_{n_{0}}\left(K_{s}\right)$, where $K_{i}=G F\left(p^{k_{i}}\right), i=1, \ldots, s$. Let $e_{i}$ denote the identity of $M_{n_{i}}\left(K_{i}\right)$. By McDonald [5, Theorem 7.12], $e_{1}, \ldots, e$, can be lifted to orthogonal idempotents $f_{1}, \ldots, f_{0}$ in $R$ with $f_{1}+\ldots+f_{0}=1$. In case $s=1, \operatorname{Rad}(R) / \operatorname{Rad}(R)^{2}$ is a nonzero right $M_{n_{1}}\left(K_{1}\right)$-module, and hence is a direct sum of simple right $M_{n_{1}}\left(K_{1}\right)$-modules. It is well known that any simple right $M_{n_{1}}\left(K_{1}\right)$-module is isomorphic to the right ideal $I$ consisting of all matrices with only the first row different from zero. Since $|I|=p^{n_{1} k_{1}}$, we see

$$
p^{n_{1} k_{1}} \leqslant\left|\operatorname{Rad}(R) / \operatorname{Rad}(R)^{2}\right| \leqslant|\operatorname{Rad}(R)|=p^{m},
$$

whence $n_{1} k_{1} \leqslant m$. Therefore

$$
|R|=p^{k_{1} n_{1}^{2}+m} \leqslant p^{m^{2}+m+1} .
$$

Now suppose $s>1$. We shall define a graph $G=(V, E)$ as follows: $V=\{1,2, \ldots, s\}$ and two distinct vertices $i$ and $j$ are joined by an edge $(i, j)$ if either $f_{i} R f_{j} \neq 0$ or $f_{j} R f_{i} \neq 0$. According to the proof of Mainwaring and Pearson [4, Theorem], 
this graph $G$ is connected. Since $\oplus_{i \neq j} f_{i} R f_{j}$ is contained in $\operatorname{Rad}(R)$, we see that $\prod_{i \neq j}\left|f_{i} R f_{j}\right| \leqslant p^{m}$. We shall estimate $\left|f_{i} R f_{j}\right|$. For the sake of simplification, we let $J=\operatorname{Rad}(R)$ and $F=G F(p)$. Now assume $f_{i} R f_{j} \neq 0$. Then $f_{i} R f_{j}$ is a nonzero $\left(f_{i} R f_{i}, f_{j} R f_{j}\right)$-bimodule. Let $M$ denote the factor module of $f_{i} R f_{j}$ by $f_{i} J f_{i} R f_{j}+f_{i} R f_{j} J f_{j}$. By virtue of Nakayama's lemma [5, Theorem 5.2], $M$ is a nonzero $\left(f_{i} R f_{i} / f_{i} J f_{i}, f_{j} R f_{j} / f_{j} J f_{j}\right)$-bimodule. Since $f_{h} R f_{h} / f_{h} J f_{h}$ is isomorphic to $M_{n_{h}}\left(K_{h}\right), 1 \leqslant h \leqslant s, M$ can be viewed as a nonzero $\left(M_{n_{i}}\left(K_{i}\right), M_{n_{j}}\left(K_{j}\right)\right)$. bimodule. Since the opposite ring of $M_{n_{j}}\left(K_{j}\right)$ is isomorphic to $M_{n_{j}}\left(K_{j}\right)$ itself, $M$ can be regarded as a nonzero left $M_{n_{i}}\left(K_{i}\right) \otimes_{F} M_{n_{j}}\left(K_{j}\right)$-module. By the way, we can easily see that $K_{i} \otimes_{F} K_{j}=G F\left(p^{k_{i}}\right) \otimes_{F} G F\left(p^{k_{j}}\right) \simeq G F\left(p^{\ell}\right)^{(d)}$, the direct sum of $d$ copies of $G F\left(p^{\ell}\right)$, where $d=\operatorname{gcd}\left\{k_{i}, k_{j}\right\}$ and $\ell=\operatorname{lcm}\left\{k_{i}, k_{j}\right\}$. Therefore $M_{n_{i}}\left(K_{i}\right) \otimes_{F} M_{n_{j}}\left(K_{j}\right) \simeq M_{n_{i} n_{j}}\left(G F\left(p^{\ell}\right)\right)^{(d)}$, whence we have $\left|f_{i} R f_{j}\right| \geqslant|M| \geqslant p^{n_{i} n_{j}}$. Consequently we obtain

$$
\sum_{(i, j) \in E} n_{i} n_{j}\left(\operatorname{lcm}\left\{k_{i}, k_{j}\right\}\right) \leqslant m
$$

Define a mapping $\psi: E \rightarrow Z$ by $\psi(i, j)=n_{i} n_{j}\left(\operatorname{lcm}\left\{k_{i}, k_{j}\right\}\right)$ for any $(i, j) \in E$. Then (1) can be rewritten as follows.

$$
\sum_{x \in E} \psi(x) \leqslant m
$$

By Lemma 1 there exists an injective mapping $\varphi: V^{\prime}=\{1,2, \ldots, s-1\} \rightarrow E$ satisfying that, for each $i \in V^{\prime}, \varphi(i)=(i, j)$ for some $j \in V$ and there exists $h \in V^{\prime}$ with $\varphi(h)=(h, s)$. Since $\left(n_{h}^{2}-1\right)\left(n_{s}^{2}-1\right) \geqslant 0$, we have

$$
\begin{aligned}
(\psi(\varphi(h)))^{2}=n_{h}^{2} n_{\lrcorner}^{2}\left(\operatorname{lcm}\left\{k_{h}, k_{\lrcorner}\right\}\right)^{2} & \geqslant\left(n_{h}^{2} k_{h}\right)\left(n_{\lrcorner}^{2} k_{\lrcorner}\right) \\
& \geqslant n_{h}^{2} k_{h}+n_{\lrcorner}^{2} k_{\triangleleft}-1 .
\end{aligned}
$$

On the other hand, by the definitions of $\varphi$ and $\psi,(\psi(\varphi(i)))^{2} \geqslant n_{i}^{2} k_{i}, 1 \leqslant i \leqslant s-1$. By the inequality (2), we have $m \geqslant \sum_{i=1}^{-1} \psi(\varphi(i))$. Hence we obtain

$$
\begin{aligned}
m^{2} & \geqslant \sum_{j=1}^{h-1}(\psi(\varphi(i)))^{2}+(\psi(\varphi(h)))^{2}+\sum_{j=h+1}^{-1}(\psi(\varphi(j)))^{2} \\
& \geqslant \sum_{i=1}^{h-1} n_{i}^{2} k_{i}+\left(n_{h}^{2} k_{h}+n_{,}^{2} k_{s}-1\right)+\sum_{j=h+1}^{:-1} n_{j}^{2} k_{j} \\
& =\sum_{i=1}^{\dot{1}} n_{i}^{2} k_{i}-1 .
\end{aligned}
$$


Therefore we have

$$
|R|=p^{n_{1}^{2} k_{1}+\ldots+n_{\theta}^{2} k_{s}+m} \leqslant p^{m^{2}+m+1} .
$$

By the above argument, the equality holds if and only if either $s=2, k_{1}=k_{2}=1$, $n_{1}=1, n_{2}=m$ or $s=2, k_{1}=k_{2}=1, n_{1}=m, n_{2}=1$. Without loss of generality, we may assume that the former occurs. In this case, we have either $\left|f_{1} R f_{2}\right|=p^{m}$ or $\left|f_{2} R f_{1}\right|=p^{m}$. If $\left|f_{1} R f_{2}\right|=p^{m}$, then $R=f_{1} R f_{1} \oplus f_{1} R f_{2} \oplus f_{2} R f_{2}, f_{1} R f_{1}=G F(p)$ and $f_{2} R f_{2}=M_{m}(G F(p))$. Hence we have

$$
R=\left(\begin{array}{cc}
f_{1} R f_{1} & f_{1} R f_{2} \\
0 & f_{2} R f_{2}
\end{array}\right) \simeq A_{m+1}(p)
$$

Similarly, if $\left|f_{2} R f_{1}\right|=p^{m}$, then $R$ is anti-isomorphic to $A_{m+1}(p)$. This completes the proof.

COROLlaRY 1. If $R$ is a finite ring not having simple ring direct summands, then $|R| \leqslant n^{n+1}$, where $n=|\operatorname{Rad}(R)|$.

Proof: First we assume that $R$ is directly indecomposable. Then $|\operatorname{Rad}(R)|=p^{m}$ for some prime $p$ and some positive integer $m$. Then we have $|R| \leqslant p^{m^{2}+m+1}$ by Theorem 1 . Since $p \geqslant 2$, we can easily see that $m^{2}+m+1 \leqslant m\left(p^{m}+1\right)$, whence we have $p^{m^{2}+m+1} \leqslant n^{n+1}$.

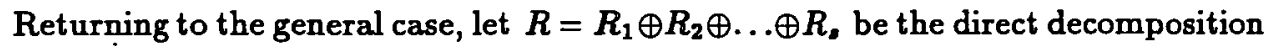
of $R$ into directly indecomposable components and let $n_{i}=\left|\operatorname{Rad}\left(R_{i}\right)\right|$. By hypothesis, each $n_{i}$ is greater than 1 , and $n=n_{1} n_{2} \ldots n_{a}$. By the result proved above, we obtain $\left|R_{i}\right| \leqslant n_{i}^{n_{i}+1}, 1 \leqslant i \leqslant s$. Hence we obtain

$$
|R|=\left|R_{1}\right| \ldots\left|R_{s}\right| \leqslant n_{1}^{n_{1}+1} \ldots n_{s}^{n_{s}+1} \leqslant n^{n+1} .
$$

As an immediate consequence of Corollary 1 , we have

Proposition 1. For a given nilpotent ring $N$ there are up to isomorphism only finitely many finite rings not having simple ring direct summands, with radical isomorphic to $N$.

Let $R^{*}$ denote the group of units of a ring $R$. Farahat [1] considered the proportion $\delta(R)=\left|R^{*}\right| /|R|$ for a finite ring $R$. We shall study $\delta(R)$ of a directly indecomposable non-simple finite ring $R$. To state the result, we need to introduce a class of rings. Let $\sigma$ be an automorphism of $G F\left(p^{m}\right)$. We denote the subring

$$
\left\{\left(\begin{array}{cc}
a & b \\
0 & \sigma(a)
\end{array}\right) \mid a, b \in G F\left(p^{m}\right)\right\}
$$

of $M_{2}\left(G F\left(p^{m}\right)\right)$ by $B_{\sigma}\left(p^{m}\right)$. 
TheOREM 2. Let $R$ be a directly indecomposable finite ring and suppose $|\operatorname{Rad}(R)|$ $=p^{m}$ where $p$ is a prime and $m$ is a positive integer. Then

$$
(1-1 / p)^{m+1} \leqslant \delta(R) \leqslant 1-1 / p^{m} .
$$

The first equality holds if and only if $R$ is isomorphic to either $G R\left(p^{2}, m\right)$ or $B_{\sigma}\left(p^{m}\right)$. The second equality holds if and only if $R$ is an algebra over $G F(p)$ such that $R / \operatorname{Rad}(R) \simeq G F(p)^{(m+1)}$.

Proof: As in the proof of Theorem 1, let $R / \operatorname{Rad}(R)=M_{n_{1}}\left(K_{1}\right) \oplus \ldots \oplus M_{n_{1}}\left(K_{0}\right)$, where $K_{i}=G F\left(p^{k_{i}}\right), 1 \leqslant i \leqslant s$. By $[1,(3.2)]$, we have

$$
\delta(R)=\sum_{i=1}^{\bullet} \delta\left(M_{n_{i}}\left(K_{i}\right)\right) \leqslant \delta\left(M_{n_{1}}\left(K_{1}\right)\right) .
$$

Also, by Farahat $[1,(3.6)]$, we have

$$
\delta\left(M_{n_{1}}\left(K_{1}\right)\right)=\left(1-1 / p^{k_{1}}\right)\left(1-1 / p^{2 k_{1}}\right) \ldots\left(1-1 / p^{n_{1} k_{1}}\right) .
$$

This is not greater than $1-1 / p^{m}$, because $k_{1} \leqslant m$ as shown in the proof of Theorem 1. Hence we obtain $\delta(R) \leqslant 1-1 / p^{m}$. The equality holds if and only if $s=n_{1}=1$ and $k_{1}=m$, that is, $R / \operatorname{Rad}(R)=G F\left(p^{m}\right)$. We determine such a ring $R$. To do this, assume that $R$ is of characteristic $p^{t}$. Then by Raghavendran [6, Theorem 8 (i)], $R$ contains a subring $S$ which is isomorphic to $G R\left(p^{t}, m\right)$. Since

$$
p^{t m}=|S| \leqslant|R|=|R / \operatorname{Rad}(R)||\operatorname{Rad}(R)|=p^{2 m}
$$

either $t=1$ or $t=2$. If $t=2$, then $R \simeq G R\left(p^{2}, m\right)$. Now suppose $t=1$. Since $\operatorname{Rad}(R) / \operatorname{Rad}(R)^{2}$ is a nonzero vector space over $R / \operatorname{Rad}(R)=G F\left(p^{m}\right)$, $\left|\operatorname{Rad}(R) / \operatorname{Rad}(R)^{2}\right| \geqslant p^{m}$. Since $|\operatorname{Rad}(R)|=p^{m}$ by hypothesis, this implies $\operatorname{Rad}(R)^{2}=0$. Then $R \simeq B_{\sigma}\left(p^{m}\right)$ for some $\sigma$ by Raghavendran [6, Theorem 3]:

Next we deal with the first inequality. We easily see

$$
\begin{aligned}
\delta(R) & =\sum_{i=1}^{\infty} \delta\left(M_{n_{i}}\left(K_{i}\right)\right) \\
& =\sum_{i=1}^{\bullet}\left\{\left(1-1 / p^{k_{i}}\right)\left(1-1 / p^{2 k_{i}}\right) \ldots\left(1-1 / p^{n_{i} k_{i}}\right)\right\} \\
& \geqslant(1-1 / p)^{n_{1}+n_{2}+\ldots+n_{4}} .
\end{aligned}
$$

From (1) in the proof of Theorem 1, we get

$$
m \geqslant \sum_{(i, j) \in E} n_{i} n_{j} .
$$


Define a mapping $\chi: E \rightarrow Z$ by $\chi(i, j)=n_{i} n_{j}$. We again employ the mapping $\varphi: V^{\prime}=\{1,2, \ldots, s-1\} \rightarrow E$ in the proof of Theorem 1. Then there exists $h \in V^{\prime}$ such that $\varphi(h)=(h, s)$. Then $\chi(\varphi(h))=n_{h} n_{,} \geqslant n_{h}+n_{,}-1$. Since $\chi(\varphi(i)) \geqslant n_{i}$, $1 \leqslant i \leqslant s-1$, we see

$$
\begin{aligned}
m & \geqslant \sum_{x \in E} \chi(x) \\
& \geqslant \sum_{i=1}^{i-1} \chi(\varphi(i)) \\
& =\sum_{i=1}^{h-1} \chi(\varphi(i))+\chi(\varphi(h))+\sum_{i=h+1}^{i-1} \chi(\varphi(i)) \\
& \geqslant \sum_{i=1}^{h-1} n_{i}+\left(n_{h}+n_{a}-1\right)+\sum_{i=h+1}^{i-1} n_{i} \\
& =\sum_{i=1}^{\infty} n_{i}-1
\end{aligned}
$$

Combining this inequality with $(3)$, we get $\delta(R) \geqslant(1-1 / p)^{m+1}$. The equality holds if and only if $s=m+1$ and $n_{i}=k_{i}=1,1 \leqslant i \leqslant s$, so that $R / \operatorname{Rad}(R) \simeq G F(p)^{(m+1)}$. By the definition of $E$, for any $(i, j) \in E$, either $f_{i} R f_{j} \neq 0$ or $f_{j} R f_{i} \neq 0$. Since $|E| \geqslant|V|-1=m$ and since

$$
\prod_{(i, j) \in E}\left\{\left|f_{i} R f_{j}\right|\left|f_{j} R f_{i}\right|\right\} \leqslant|\operatorname{Rad}(R)|=p^{m}
$$

we conclude that $|E|=m$ and $\left|f_{i} R f_{j}\right|\left|f_{j} R f_{i}\right|=p$ for each $(i, j) \in E$. Since $R=$ $\bigoplus_{i, j=1}^{m+1} f_{i} R f_{j}$ as additive group, this implies $\operatorname{char}(R)=p$. Therefore $R$ is a $(2 m+1)$ dimensional algebra over $G F(p)$. This completes the proof.

We shall give an example of a ring satisfying the second equality in Theorem 2.

EXAMPLE. Let $e_{i j}$ be the standard matrix units in $M_{m+1}(G F(p))$ and consider the subalgebra

$$
R=\sum_{i=1}^{m+1} G F(p) e_{i i}+\sum_{j=2}^{m+1} G F(p) e_{1 j}
$$

Then $R$ is a directly indecomposable ring such that $|\operatorname{Rad}(R)|=p^{m}$ and $\delta(R)=$ $1-1 / p^{m}$.

Note that $1+\operatorname{Rad}(R)$ is a (normal) subgroup of the group $R^{*}$, so that $|\operatorname{Rad}(R)| \leqslant$ $\left|R^{*}\right|$. Thus the following improves Stewart [7, Corollary 2.5] in case $R$ is a directly indecomposable finite $2^{\prime}$-ring with nonzero radical. 
COROLlaRY 2. If $R$ is a directly indecomposable non-simple finite $\mathbf{2}^{\prime}$-ring, then $|R|<\left|R^{*}\right||\operatorname{Rad}(R)|$.

Proof: By hypotheses, $|\operatorname{Rad}(R)|=p^{m}$ for some prime $p>2$ and some positive integer $m$. By Theorem 2 we have

$$
|R| \leqslant\left(\frac{p}{p-1}\right)^{m+1}\left|R^{*}\right|
$$

Now, since $p>2$, we have

$$
\left(\frac{p}{p-1}\right)^{m+1}<p^{m}=|\operatorname{Rad}(R)| .
$$

\section{REFERENCES}

[1] H.K. Farahat, 'The multiplicative groups of a ring', Math. Z. 87 (1965), 378-384.

[2] F.J. Flanigan, 'Radical behavior and the Wedderburn family', Bull. Amer. Math. Soc. 79 (1973); 66-70.

[3] M. Hall, 'The position of the radical in an algebra', Trans. Amer. Math. Soc. 48 (1940), 391-404.

[4] D. Mainwaring and K.R. Pearson, 'Decomposability of finite rings', J. Austral. Math. Soc. Ser. A 28 (1979), 136-138.

[5] B.R. McDonald, Finite rings with identity (Marcel Dekker, New York, 1974).

[6] R. Raghavendran, 'Finite associative rings', Compositio Math. 21 (1969), 195-229.

[7] I. Stewart, 'Finite rings with a specified group of units', Math. Z. 126 (1972), 51-58.

Department of Mathematics

Okayama University

Okayama 700

Japan
Department of Mathematics

Aichi Institute of Technology

Yakusa-chô, Toyota, 470-03

Japan 